\title{
Role of endothelial nitric oxide synthase gene polymorphisms in predicting aneurysmal subarachnoid hemorrhage in South Indian patients
}

\author{
Linda Koshy $^{\mathrm{a}}$, H.V. Easwer ${ }^{\mathrm{b}}$, N.V. Neetha ${ }^{\mathrm{a}}$, Chandrasekhar Natarajan ${ }^{\mathrm{a}}$, R.N. Bhattacharya ${ }^{\mathrm{b}}$ and \\ Moinak Banerjee ${ }^{\mathrm{a}, *}$ \\ ${ }^{a}$ Human Molecular Genetics Laboratory, Rajiv Gandhi Centre for Biotechnology, Thiruvananthapuram, Kerala, \\ India \\ ${ }^{\mathrm{b}}$ Sree Chitra Tirunal Institute for Medical Science and Technology, Thiruvananthapuram, Kerala, India
}

\begin{abstract}
Endothelial nitric oxide synthase $(e N O S)$ gene polymorphisms have been implicated as predisposing genetic factors that can predict aneurysmal subarachnoid hemorrhage (aSAH), but with controversial results from different populations. Using a case-control study design, we tested the hypothesis whether variants in $e N O S$ gene can increase risk of aSAH among South Indian patients, either independently, or by interacting with other risk factors of the disease. We enrolled 122 patients, along with 224 ethnically matched controls. We screened the intron-4 27-bp VNTR, the promoter T-786C and the exon-7 G894T SNPs in the eNOS gene. We found marked interethnic differences in the genotype distribution of $e N O S$ variants when comparing the South Indian population with the reported frequencies from Caucasian and Japanese populations. Genotype distributions in control and patient populations were found to be in Hardy-Weinberg equilibrium. In patients, the allele, genotype and estimated haplotype frequencies did not differ significantly from the controls. Multiple logistic regression indicated hypertension and smoking as risk factors for the disease, however the risk alleles did not have any interaction with these risk factors. Although the eNOS polymorphisms were not found to be a likely risk factor for aSAH, the role of factors such as ethnicity, gender, smoking and hypertension should be evaluated cautiously to understand the genotype to phenotype conversion.
\end{abstract}

Keywords: eNOS, SNP, aneurysm, subarachnoid hemorrhage, India

\section{Introduction}

Intracranial aneurysm is a fairly common condition that is often asymptomatic until the time of rupture, resulting in aneurysmal subarachnoid hemorrhage (aSAH), ensuing significant morbidity and mortality. It is the cause for an estimated five to 15 percent of

*Corresponding author: Moinak Banerjee, Ph.D, Human Molecular Genetics Laboratory, Rajiv Gandhi Centre for Biotechnology, Thycaud P.O., Thiruvananthapuram-695014, Kerala, India. Tel.: +91 471 2341716; Fax: +91 471 2348096; E-mail: moinak_ban@hotmail.com; mbanerjee@rgcb.res.in. all strokes, with an overall incidence of 16 to 22 per 100,000 population [2]. The reported prevalence rate of intracranial aneurysms in India varies from $0.75 \%$ to $10.3 \%$ as determined in angiographic and autopsy studies $[9,18]$. Current evidence supports the concept that the pathogenesis of intracranial aneurysms has a multifactorial origin, where the essential defect of the arterial wall may, at least in part be genetically determined [19].

Endothelial nitric oxide synthase (eNOS) expressed on endothelial cells plays a major role in shear-stress response related endothelial dysfunction, which has been recognized as the underlying pathological process of 
intracranial aneurysms [22]. Endothelium derived nitric oxide (NO) synthesized by nitric oxide synthase has a powerful vasodilatory action and studies on the response of NO levels to shear-stress investigated in human cerebral endothelial cells has indicated a linear relationship [15]. In addition, it has been demonstrated that as blood vessels age, shear-stress induced eNOS becomes impaired leading to decreased NO bioavailability and endothelial dysfunction, which is likely to contribute to age-associated vascular stiffness [21]. A rat model of SAH used to demonstrate that cerebral microvessels have endothelial dysfunction, has revealed predisposition to vasoconstriction and spasm, suggesting impaired translation of eNOS [17]. Interestingly, aSAH is a late onset disorder and therefore reduced NO bioavailability due to eNOS expression variants could account for the etiology of aSAH.

Located on chromosome 7q35-36, eNOS has three well-defined polymorphisms; the T-786C SNP (rs2070744) in the promoter region, the 27-bp VNTR in intron 4 and the G894T SNP (rs1799983) in exon 7. These gene mutations have been shown to increase susceptibility either singly, or in combination with conditions that includes hypertension, stroke, cerebral smallvessel disease, and abdominal aortic aneurysms [1,6, $8,16]$. It has been demonstrated that the eNOS gene is functionally polymorphic, where some of the allelic variants may constitutively express altered phenotypic expression. These allelic variants of the gene, can result in decreased NO production, which may be explained either in terms of functional or quantitative deficiency (abnormalities of transcription, mRNA stability, formation of catalytically defective protein) or by enhanced degradation of the enzyme [4]. The eNOS gene variants with altered eNOS regulation might render the blood vessels susceptible to demographic factors.

Recently, a study done in a North American population had analyzed the role of these three eNOS gene polymorphisms as potential risk factors that can predict susceptibility to aSAH and cerebral vasospasm [10]. They had reported a positive association with regard to the 4a allele of the eNOS 27-bp VNTR polymorphism $(P=0.007)$, where they found an odds of approximately 3.95 for being an aSAH case for those persons with atleast one $4 \mathrm{a}$ allele. In the present study, we have explored the hypothesis of whether polymorphisms in the eNOS gene are associated with aSAH in South Indian population, in relation to its other phenotypic variables and ethnicity.

\section{Materials and methods}

\subsection{Study population}

We studied 122 unrelated patients who had been consecutively admitted to the Department of Neurosurgery at Sree Chitra Tirunal Institute for Medical Science and Technology (SCTIMST), Kerala. The patient group consisted of 70 males and 52 females. Patients with aSAH were defined by symptoms suggestive of SAH combined with subarachnoid blood on computed tomography and a proven aneurysm. All patients had angiographically documented saccular aneurysms with SAH confirmed by cranial computed tomography (CT), magnetic resonance angiography (MRA) and digital subtraction angiography (DSA). The neurological grade was classified by the World Federation of Neurological Surgeons (WFNS) scale and all grades of $\mathrm{SAH}$ were eligible for inclusion. All preexisting medical conditions were documented. Exclusion criteria included nonsaccular aneurysm, arteriovenous malformations and haematological disorders. Demographic variables of aSAH patients in the South Indian population are shown in Table 1. The control group consisted of 224 unrelated, age and ethnically-matched volunteers recruited from individuals admitted to the hospital for any reason other than neurological diseases. This group comprised of 110 males and 114 females. All subjects were of the same ethnic origin and all participants gave informed consent for the study. The outline of the present study was approved by the Institutional Ethical Committee for Biomedical Subjects.

\subsection{Genotyping of the eNOS polymorphisms}

Peripheral venous blood from each participant was drawn and used for the isolation of genomic DNA using the standard organic extraction protocol. Presence of the T-786C and G894T SNPs were determined by polymerase chain reaction followed by restriction enzyme (RE) digestion. The 27-bp VNTR was genotyped as described previously [25]. The PCR primers and annealing temperatures are summarized in Table 2. PCR assays was carried out in a $20 \mu \mathrm{l}$ volume with 100ng of genomic DNA, $10 \mathrm{pM}$ of each primer, $2.5 \mathrm{mM}$ dNTP (Amersham), $1.5 \mathrm{mM} \mathrm{MgCl}_{2}$ and 10x PCR buffer [50 mM KCl, $500 \mathrm{mM}$ Tris buffer, $160 \mathrm{mM}$ $\left(\mathrm{NH}_{4}\right)_{2} \mathrm{SO}_{4}, \mathrm{pH} 8.8$, and $0.1 \%$ Tween 20], $0.1 \%$ Triton $\mathrm{X}-100$ and $0.5 \mathrm{U}$ Taq polymerase (Sigma). After a hot start at $95^{\circ} \mathrm{C}$ for 4 minutes, amplification was achieved by 35 cycles of denaturation at $94^{\circ} \mathrm{C}$ for 30 seconds, 
Table 1

Demographic and clinical characteristics of study subjects

\begin{tabular}{lll}
\hline Characteristic(s) & Patient Group & Control Group \\
\hline Total no. of subjects & 122 & 224 \\
Mean age (range), years & $51.49 \pm 11.4$ years & $46.71 \pm 14.1$ years \\
Male, n & 70 & 110 \\
Mean age (range), years & $48.9(18-71)$ years & 46.7 (32-87) years \\
Female, n & 52 & 114 \\
Mean age (range), years & $54.98(20-75)$ years & 46.5 (31-81) \\
Hypertension & 42 & 18 \\
Cigarette smoking & 56 & 23 \\
Alcohol consumption & 23 & 21 \\
Diabetes mellitus & 7 & 29 \\
Admission WFNS grade, n & & $\mathrm{n} / \mathrm{a}$ \\
I & 90 & \\
II & 17 & \\
III & 7 & \\
IV & 7 & \\
V & 1 & \\
Location, n & & \\
Internal carotid artery & 28 & \\
Middle cerebral artery & 26 & \\
Anterior communicating artery & 56 & \\
Anterior cerebral artery & 11 & \\
Posterior communicating artery & 12 & \\
Basilar artery & 5 & \\
Multiple, n & 17 & \\
Two aneurysms & 14 & \\
Three aneurysms & 3 & \\
\hline
\end{tabular}

Table 2

Primer sequences

\begin{tabular}{llccc}
\hline Polymorphism & Primer pairs Sense/Antisense & Ta, $\left({ }^{\circ} \mathrm{C}\right)$ & $\mathrm{RE}$ & Digestion products, bp \\
\hline G894T & 5'-AAGGCAGGAGACAGTGGATGGA-3' & 60 & BanII & $248,163,85$ \\
& 5'-CCCAGTCAATCCCTTTGGTGCTCA-3' & & & \\
T-786C & 5'-GCATGCACTCTGGCCTGAAGT-3' & 60 & MspI & $162,116,61,46$ \\
& 5'-CAGGAAGCTGCCTTCCAGTGC-3' & & & \\
27-bp VNTR & 5'-AGGCCCTATGGTAGTGCCTTT-3' & 56 & $\ldots$ & 420,393 \\
& 5'-TCTCTTAGTGCTGTGGTCAC-3' & & & \\
\hline
\end{tabular}

annealing at specific temperatures, see Table 2, for 30 seconds, and extension at $72^{\circ} \mathrm{C}$ for 30 seconds, by using a thermal cycler (Eppendorff). PCR products were restriction digested with $\mathrm{MspI}$ and BanII restriction endonucleases (New England Biolabs), respectively. Restriction digested products were resolved using $3 \%$ Nusieve agarose gel and visualized under UV light after ethidium bromide staining. All three genotypes of the T-786C and G894T SNPs were further verified by direct sequencing using ABI PRISM BigDye Terminators v3.1 Cycle Sequencing Kit (Applied Biosystems), and analyzed using ABI PRISM 3730 Genetic Analyzer (Applied Biosystems).

\subsection{Statistical analysis and haplotype construction}

Deviations from the Hardy-Weinberg equilibrium were tested for all polymorphisms in cases and con- trols by comparing observed and expected genotype frequencies with an exact goodness of fit test. Odds ratios, as the estimates of relative risk of disease, with 95\% confidence intervals were calculated to compare allele and genotype frequencies using chi-square analysis. Multiple logistic regression analysis was performed with the diagnosis as the dependent variable, and covariates such as age at onset, age above 50 years, female gender, smoking, hypertension, diabetes mellitus, alcohol use, and the eNOS genotypes as the independent variables. For analyzing dominant inheritance model (e.g., eNOS 786CC+TC vs TT), genotypes were coded as 1 (heterozygous or homozygous for the minor allele) or 0 (the major allele homozygote). Regression analysis was conducted using the program SPSS 13.0 for Windows (SPSS Inc, Chicago, IL, USA) statistical analysis software. Additionally, the interaction of genotypes with the various non-genetic risk factors was 
Table 3

Allele and genotype frequencies of $e N O S$ polymorphisms in controls and cases

\begin{tabular}{llllll}
\hline Polymorphism & Controls, $\mathrm{n}(\%)$ & aSAH, $\mathrm{n}(\%)$ & $P$-value & OR & $95 \% \mathrm{CI}$ \\
\hline T-786C SNP & & & & & \\
T/T & $136(60.7)$ & $68(55.7)$ & 0.37 & 1.23 & 0.79 to 1.92 \\
T/C & $81(36.2)$ & $51(41.8)$ & & & \\
C/C & $7(3.1)$ & $3(2.5)$ & & & \\
$\quad$ allele C & $95(21.2)$ & $57(23.4)$ & 0.51 & 1.13 & 0.78 to 1.65 \\
27-bp VNTR & & & & & \\
4b/4b & $143(63.8)$ & $77(63.1)$ & 0.89 & 1.03 & 0.65 to 1.63 \\
4a/4b & $77(34.4)$ & $40(32.8)$ & & & \\
4a/4a & $4(1.8)$ & $5(4.1)$ & & & \\
allele 4a & $85(19.0)$ & $50(20.5)$ & 0.63 & 1.10 & 0.75 to 1.63 \\
G894T SNP & & & & & \\
G/G & $159(71.0)$ & $85(69.7)$ & 0.80 & 1.07 & 0.66 to 1.72 \\
G/T & $61(27.2)$ & $35(28.7)$ & & & \\
T/T & $4(1.8)$ & $2(1.6)$ & & & \\
allele T & $69(15.4)$ & $39(16.0)$ & 0.84 & 1.05 & 0.68 to 1.60 \\
\hline
\end{tabular}

studied using the Crosstab option of the statistical analysis software program SPSS 13.0 (SPSS Inc, Chicago, IL). Haplotype and pairwise linkage disequilibrium analyses (LD) were performed by COCAPHASE program of the UNPHASED software suite, in which the expectation-maximization (EM) algorithm is used to estimate frequencies [5]. The extent of LD was expressed in terms of the maximum likelihood estimate of disequilibrium, $D^{\prime}$ and viewed in HAPLOVIEW. The power of the present study was calculated using the allele frequencies reported earlier for the 27-bp VNTR polymorphism, at a 5\% test level (alpha $=0.05$, two tailed) [10]. The statistical power of the study was calculated as described by Cohen [3]. Probability $(P)$ values of less than 0.05 were considered statistically significant.

\section{Results}

The observed allele and genotype frequencies for T-786C and G894T SNPs, and intron 27-bp VNTR polymorphism is presented in Table 3 . The observed genotype frequencies did not deviate from the HardyWeinberg equilibrium in both patients and control subjects. No significant differences in the distribution of allele and genotype frequencies in patient and control groups were observed (Table 3). We tested the LD between all the pairwise polymorphisms and observed that the T-786C and 27-bp VNTR loci in both patient and control groups showed linkage, $\left(D^{\prime}=0.6033\right.$ in controls and $D^{\prime}=0.6247$ in patients). However, we found a negative $D^{\prime}$ value $(-1.00$ in controls and -0.4117 in patients) for the association between the variants in the G894T and in 27-bp VNTR loci, indi-
Table 4

Estimated eNOS haplotype frequencies of aSAH patients and controls

\begin{tabular}{lccc}
\hline Haplotype & aSAH freq & Control freq & $P$-value \\
\hline T-4b-G & 0.6027 & 0.6134 & 0.673 \\
T-4b-T & 0.1028 & 0.1155 & 0.779 \\
T-4a-G & 0.0607 & 0.0479 & 0.715 \\
T-4a-T & $<0.0001$ & 0.0112 & 0.243 \\
C-4b-G & 0.0359 & 0.0541 & 0.497 \\
C-4b-T & 0.0537 & 0.0273 & 0.197 \\
C-4a-G & 0.1407 & 0.1306 & 0.590 \\
C-4a-T & 0.0034 & $<0.0001$ & 0.768 \\
\hline
\end{tabular}

cating that the rarer variant $4 \mathrm{a}$ in intron 4 is associated with the wild-type variant Glu298 in exon 7 or vice versa. Comparison of haplotypes frequencies revealed no single haplotype overrepresented in aSAH patients (Table 4). T-4a-G was observed to be the most frequent haplotype and was observed in more than $60 \%$ of both the case and control populations.

The previously reported risk genotype, $4 \mathrm{a} / 4 \mathrm{~b}$ of 27 bp VNTR polymorphism was not significantly associated with an increased risk of developing aSAH. Likewise, the T-786C SNP, which was in strong linkage disequilibrium with the 27-bp VNTR, was again not significantly associated with aSAH. After controlling for age and sex multiple logistic regression analysis showed a lack of association of genotypes of the eNOS polymorphisms with aSAH. Non-genetic risk factors such as hypertension and smoking were identified as independent predictors of aSAH $(P=0.0001$ for the entire model), as shown in Table 5. However, no significant interactions were observed between the risk alleles and the non-genetic risk factors.

The power calculation for the 27-bp VNTR $4 a / 4 b$ allele was done as follows. The power required in this study to detect the specified difference in the frequen- 
Table 5

Multiple logistic regression analysis evaluating the odds ratios of eNOS polymorphisms for aSAH according to dominant model

\begin{tabular}{lrlr}
\hline Variables & OR & $95 \%$ C.I & $P$-value \\
\hline Age at onset & 1.007 & $0.96-1.05$ & 0.754 \\
Age above 50 yrs & 1.846 & $0.55-6.25$ & 0.324 \\
Female sex & 1.373 & $0.60-3.16$ & 0.455 \\
Diabetes mellitus & 0.271 & $0.08-0.95$ & 0.042 \\
Hypertension & 7.327 & $2.54-21.17$ & $<0.001$ \\
Smoking habit & 12.864 & $3.90-42.41$ & $<0.001$ \\
Alcohol use & 0.377 & $0.11-1.32$ & 0.128 \\
eNOS 786TC+CC & 1.055 & $0.42-2.63$ & 0.909 \\
eNOS 4ab+4aa & 0.621 & $0.24-1.63$ & 0.333 \\
eNOS 894GT+TT & 0.909 & $0.42-1.98$ & 0.811 \\
\hline
\end{tabular}

cy of the 27-bp VNTR 4a/4b allele was calculated as $p_{\mathrm{SAH}}=0.265$ and $p_{\mathrm{C}}=0.133$, the two frequencies of the VNTR $4 \mathrm{a}$ allele in patients with aSAH and controls, respectively, reported in a previous study [10]. Using the formula as described by Cohen, we obtained $\mathrm{Z}_{1-\beta}=2.54$. This gives the value of $t$-statistic to be referred to the table of normal distribution to obtain the power, $1-\beta=0.994$.

\section{Discussion}

In the present study we were unable to observe any significant variation in the eNOS polymorphisms at the allele, genotype or haplotype levels, in South Indian patients and controls. Although a strong rationale for genetic variants in the eNOS gene contributing to reduced NO bioavailability in cerebral blood vessels had been proposed for the increased risk for aSAH, our results could not substantiate this. Initial studies done in a North American population of Caucasian origin had suggested the role of these three eNOS gene polymorphisms as risk factors that could predict susceptibility to aSAH, and therefore, suggested its use as a diagnostic marker [10]. It had been reported that heterozygosity for the eNOS 27-bp VNTR polymorphism was almost three times more prevalent in cases $(49 \%)$ than controls (18\%), $P=0.002$. The eNOS T-786C SNP was also shown to significantly differentiate between the presence and severity of post-SAH cerebral vasospasm in Caucasian patients, $P=0.04$. However, subsequent case-control studies in European, Japanese and Korean populations provided contradictory results $[12,13$, 20]. Although these studies indicated ethnic variability in the distribution of risk alleles, the non-genetic risk factors associated with aSAH had not been critically examined.
The impact of ethnicity on the distribution of these clinically relevant $e N O S$ gene polymorphisms had been effectively studied in Caucasian, African-American and Asian populations [23]. Likewise, we also observed significant allelic and genotypic variability when compared to different study populations (Table 6). The 4 a risk allele frequency of $e N O S$ 27-bp VNTR in our study was comparable to the European population while it was least frequent allele in Japanese population. Though the risk genotype $4 \mathrm{a} / 4 \mathrm{~b}$ was significantly higher in South Indian patients when compared to North American patients, it did not manifest its debilitating phenotype. The eNOS $-786 \mathrm{C}$ risk allele had a lower frequency in the South Indian population $(21.4 \%)$ in comparison to the Caucasians of North America and Europe, while in the heterozygous genotypic combination the $-786 \mathrm{C}$ allele was comparable to these populations. The differences in allele frequencies among the study populations were also reflected subsequently in the haplotype frequencies. Khurana et al. reported no significant LD among the three polymorphisms [11], whereas, we found the T-786C SNP, in modest LD with the 27-bp repeat in our population. This was in agreement with the Japanese study that reported a similar linkage for these two polymorphisms [13]. Moreover, we found no significant difference in the LD pattern of cases and controls (Fig. 1), ascertaining that the eNOS gene polymorphisms can be ruled out as risk factors for the aSAH phenotype. However, the arterial walls of individuals with risk genotypes are likely to be more susceptible to environmental insults which are influenced by ethnicity and social factors.

In a complex disease, apart from the disease per se, factors and circumstances that associate with the disease have as much importance. In India, in addition to multicultural ethnicity the prevalence of risk factors such as hypertension (54.5\%), and smoking (18.4\%), that associate with aSAH are also reported to be higher than the global average burden of these individual risk factors $[7,24,26]$. The difference of our results and those of the earlier groups can be explained in terms of the different allele frequencies, shown explicitly by the varying LD patterns as well as the prevalence of risk factors in the patient groups taken from different ethnic backgrounds. The putative functions of the eNOS polymorphisms or its linked variants at other sites may be dependent on the presence of environmental risk factors. However, our observations indicate hypertension and smoking as significant independent risk factors, which do not associate with the risk genotypes. It has been reported that when the risk of disease conferred 
Table 6

Comparison of genotype and allele frequencies of eNOS polymorphisms in South Indian and other study populations

\begin{tabular}{|c|c|c|c|c|}
\hline Polymorphism & $\begin{array}{c}\text { South India } \\
\text { (present study } \\
\text { as reference) } \\
\text { n-224 }\end{array}$ & $\begin{array}{c}\text { N. America } \\
\text { (Khuranan et al. 2004) } \\
\text { n-90 }\end{array}$ & $\begin{array}{c}\text { Europe } \\
\text { (Krex et al. 2006) }\end{array}$ & $\begin{array}{c}\text { Japan } \\
\text { (Krischek et al. 2006) } \\
\text { n-176 }\end{array}$ \\
\hline \multicolumn{5}{|l|}{ T-786C SNP } \\
\hline $\mathrm{T} / \mathrm{T}$ & 0.603 & 0.310 & 0.386 & 0.820 \\
\hline $\mathrm{T} / \mathrm{C}$ & 0.366 & 0.510 & 0.467 & 0.130 \\
\hline \multirow[t]{2}{*}{$\mathrm{C} / \mathrm{C}$} & 0.031 & 0.180 & 0.147 & 0.050 \\
\hline & & $P<0.0001$ & $P<0.0001$ & $P<0.0001$ \\
\hline allele & Т 0.786 & 0.570 & 0.619 & 0.850 \\
\hline \multirow[t]{2}{*}{ allele } & C 0.214 & 0.430 & 0.381 & 0.150 \\
\hline & & $P<0.0001$ & $P<0.0001$ & $P=0.016$ \\
\hline \multicolumn{5}{|l|}{ 27-bp VNTR } \\
\hline $4 \mathrm{~b} / 4 \mathrm{~b}$ & 0.638 & 0.780 & 0.667 & 0.810 \\
\hline $4 b / 4 a$ & 0.344 & 0.180 & 0.291 & 0.170 \\
\hline \multirow[t]{2}{*}{$4 a / 4 a$} & 0.018 & 0.040 & 0.042 & 0.020 \\
\hline & & $P=0.008$ & $P=0.21$ & $P=0.0005$ \\
\hline allele $4 b$ & 0.810 & 0.870 & 0.812 & 0.900 \\
\hline \multirow[t]{2}{*}{ allele $4 \mathrm{a}$} & 0.190 & 0.130 & 0.188 & 0.100 \\
\hline & & $P=0.09$ & $P=0.94$ & $P=0.0006$ \\
\hline \multicolumn{5}{|l|}{ G894T SNP } \\
\hline $\mathrm{G} / \mathrm{G}$ & 0.710 & 0.220 & 0.505 & 0.820 \\
\hline $\mathrm{G} / \mathrm{T}$ & 0.272 & 0.610 & 0.400 & 0.130 \\
\hline \multirow[t]{2}{*}{$\mathrm{T} / \mathrm{T}$} & 0.018 & 0.170 & 0.095 & 0.050 \\
\hline & & $P<0.0001$ & $P<0.0001$ & $P=0.001$ \\
\hline allele $\mathrm{G}$ & 0.846 & 0.530 & 0.705 & 0.890 \\
\hline \multirow[t]{2}{*}{ allele $\mathrm{T}$} & 0.154 & 0.470 & 0.295 & 0.110 \\
\hline & & $P<0.0001$ & $P<0.0001$ & $P=0.076$ \\
\hline
\end{tabular}

${ }^{*} P$ value denotes individual difference between the present study and other study populations.

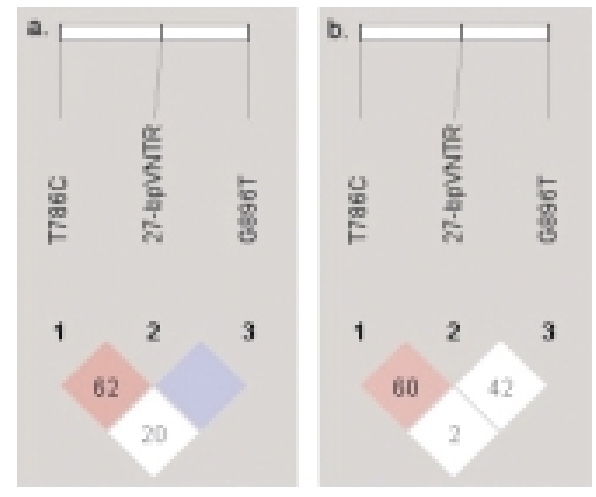

Fig. 1. LD patterns of $e N O S$ polymorphisms in cases (a) and controls (b).

by the polymorphic variant of a haemostatic gene is reduced, it could be possible that environmental risk factors are so dominant that genetic polymorphisms provide a negligible and rather insignificant contribution to the overall risk [14].

In conclusion, we could not observe any role of haploinsufficiency or homozygosity of the risk alleles of eNOS gene in causing intracranial aneurysm or aSAH, suggesting that genetic variants in the $e N O S$ gene re- sponsible for the regulation of haemodynamic stress in the arterial endothelium are unlikely to be susceptibility factors for the disease. It is however possible, that rare variants in other regions in the eNOS gene may have a role in aSAH. It is also conceivable that causative polymorphisms could be lying in genes involved in the upstream events of NO formation. Our study contradicts the seminal work on the role of $e N O S$ in aSAH in North American population but is in agreement with majority of the reports on lack of association in European and Asian populations. The study also supports that ethnic background coupled with different environmental factors might associate to aggravate the pathogenesis of the disease. However, the possibility of a multigenic combination in the NO pathway genes coupled with a genotypic tag for associated risk phenotypes such as hypertension and cigarette smoking as a causative event in the pathogenesis of aSAH cannot be ruled out.

\section{Acknowledgements}

Linda Koshy would like to acknowledge University Grants Commission, New Delhi, India, for Senior Research Fellowship. 


\section{References}

[1] N. Akar, E. Akar, S. Cin, G. Deda, F. Avcu and A. Yalcin, Endothelial nitric oxide synthase intron 4, 27 bp repeat polymorphism in Turkish patients with deep vein thrombosis and cerebrovascular accidents, Thromb Res 94 (1999), 63-64.

[2] J.B. Bederson, I.A. Awad, D.O. Weibers, D. Piepgras, E.C. Jr. Haley, T. Brott, G. Hademenos, D. Chyatte, R. Rosenwasser and C. Caroselli, Recommendations for the management of patients with unruptured intracranial aneurysms: a statement for health care professionals from the Stroke Council of the American Heart association, Stroke 31 (2000), 2742-2750.

[3] J. Cohen, Statistical Power Analysis for the Behavioral Sciences, 2nd ed., Hillsdale, Lawrence Erlbaum Associates, NJ, 1988.

[4] V.E. Dosenko, V.Y. Zagoriy, N.V. Haytovich, O.A. Gordok and A.A. Moibenko, Allelic polymorphism of endothelial NOsynthase gene and its functional manifestations, Acta Biochim Pol 53 (2006), 299-302.

[5] F. Dudbridge, Pedigree disequilibrium tests for multilocus haplotypes, Genet Epidemiol 25 (2003), 115-121.

[6] C. Fatini, F. Sofi, E. Sticchi, P. Bolli, I. Sestini, M. Falciani, L. Azas and G. Pratesi, eNOS G894T polymorphism as a mild predisposing factor for abdominal aortic aneurysm, $J$ Vasc Surg 42 (2005), 415-419.

[7] D. Gan, Diabetes Atlas, 2nd ed., Brussels, International Diabetes Federation, 2003.

[8] A. Hassan, K. Gormley, M. O’Sullivan, J. Knight, P. Sham, P. Vallance, J. Bamford and H. Markus, Endothelial nitric oxide gene haplotypes and risk of cerebral small-vessel disease, Stroke 35 (2004), 654-659.

[9] K. Kapoor and V.K. Kak, Incidence of intracranial aneurysms in north-west Indian population, Neurol India 51 (2003), 2226.

[10] V.G. Khurana, Y.R. Sohni, W.I. Mangrum, R.L. McClelland, D.J. O'Kane, F.B. Meyer and I. Meissner, Endothelial nitric oxide synthase gene polymorphisms predict susceptibility to aneurysmal subarachnoid hemorrhage and cerebral vasospasm, J Cereb Blood Flow Metab 24 (2004), 291-297.

[11] V.G. Khurana, I. Meissner, Y.R. Sohni, W.R. Bamlet, R.L. McClelland, J.M. Cunningham and F.B. Meyer, The presence of tandem endothelial nitric oxide synthase gene polymorphisms identifying brain aneurysms more prone to rupture, $J$ Neurosurg 102 (2005), 526-531.

[12] D. Krex, S. Fortun, E. Kuhlisch, H.K. Schackert and G. Schackert, The role of endothelial nitric oxide synthase (eNOS) genetic variants in European patients with intracranial aneurysms, J Cereb Blood Flow Metab 26 (2006), 1250-1255.

[13] B. Krischek, H. Kasuya, H. Akagawa, A. Tajima, A. Narita, H. Onda, T. Hori and I. Inoue, Using endothelial nitric oxide synthase gene polymorphisms to identify intracranial aneurysms more prone to rupture in Japanese patients, J Neurosurg $\mathbf{1 0 5}$ (2006), 717-722.
[14] D.A. Lane and P.J. Grant, Role of hemostatic gene polymorphisms in venous and arterial thrombotic disease, Blood 95 (2000), 1517-1532.

[15] G.A. Mashour and R.J. Boock, Effects of shear stress on nitric oxide levels of human cerebral endothelial cells cultured in an artificial capillary system, Brain Research 842 (1999), 233 238.

[16] Y. Miyamoto, Y. Saito, N. Kajiyama, M. Yoshimura, Y. Shimasaki, M. Nakayama, S. Kamitani, M. Harada, M. Ishikawa, K. Kuwahara, E. Ogawa, I. Hamanaka, N. Takahashi, T. Kaneshige, H. Teraoka, T. Akamizu, N. Azuma, Y. Yoshimasa, T. Yoshimasa, H. Itoh, I. Masuda, H. Yasue and K. Nakao, Endothelial nitric oxide synthase gene is positively associated with essential hypertension, Hypertension 32 (1998), 3-8.

[17] K.W. Park, C. Metais, H.B. Dai, M.E. Comunale and F.W. Sellke, Microvascular endothelial dysfunction and its mechanism in a rat model of subarachnoid hemorrhage, Anesth Analg 92 (2001), 990-996.

[18] B. Ramamurthi, Incidence of intracranial aneurysms in India, J Neurosurg 30 (1969), 154-157.

[19] W.I. Schievink, Genetics of intracranial aneurysms, Neurosurgery 40 (1997), 651-662.

[20] M.K. Song, M.K. Kim, T.S. Kim, S.P. Joo, M.S. Park, B.C. Kim and K.H. Cho, Endothelial nitric oxide gene T-786C polymorphism and subarachnoid hemorrhage in Korean population, J Korean Med Sci 21 (2006), 922-926.

[21] K.G. Soucy, S. Ryoo, A. Benjo, H.K. Lim, G. Gupta, J.S. Sohi, J. Elser, M.A. Aon, D. Nyhan, A.A. Shoukas and D.E. Berkowitz, Impaired shear stress-induced nitric oxide production through decreased NOS phosphorylation contributes to age-related vascular stiffness, J Appl Physiol 101 (2006), 1751-1759.

[22] W.E. Stehbens, Cerebrovascular disease, in: Pathology of the Cerebral Blood Vessels. Vascular Pathology, W.E. Stehbens and J.T. Lie, eds, Chapman \& Hall, London, 1995, pp. 437488.

[23] J.E. Tanus-Santos, M. Desai, L.R. Deak, J.C. Pezzullo, D.R. Abernethy, D.A. Flockhart and J.E. Freedman, Effects of ethnicity on the distribution of clinically relevant endothelial nitric oxide variants, Pharmacogenetics 11 (2001), 719-725.

[24] S.V. Subramanian, S. Nandy, M. Kelly, D. Gordon, G. Davey and R.S. Smith, Patterns and distribution of tobacco consumption in India: cross sectional multilevel evidence from the 1998-9 national family health survey, BMJ 328 (2004), 801-806.

[25] Y. Wang, S. Kikuchi, H. Suzuki, S. Nagase and A. Koyama, Endothelial nitric oxide synthase gene polymorphism in intron 4 affects the progression of renal failure in non-diabetic renal diseases, Nephrol Dial Transplant 14 (1999), 2898-2902.

[26] M.G. Zachariah, K.R. Thankappan, S.C. Alex, P.S. Sarma and R.S. Vasan, Prevalence, correlates, awareness, treatment, and control of hypertension in a middle-aged urban population in Kerala, Indian Heart J 55 (2003), 245-251. 


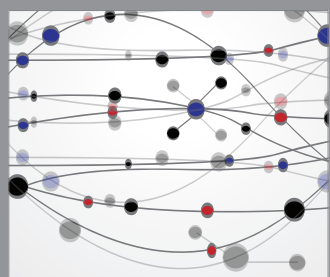

The Scientific World Journal
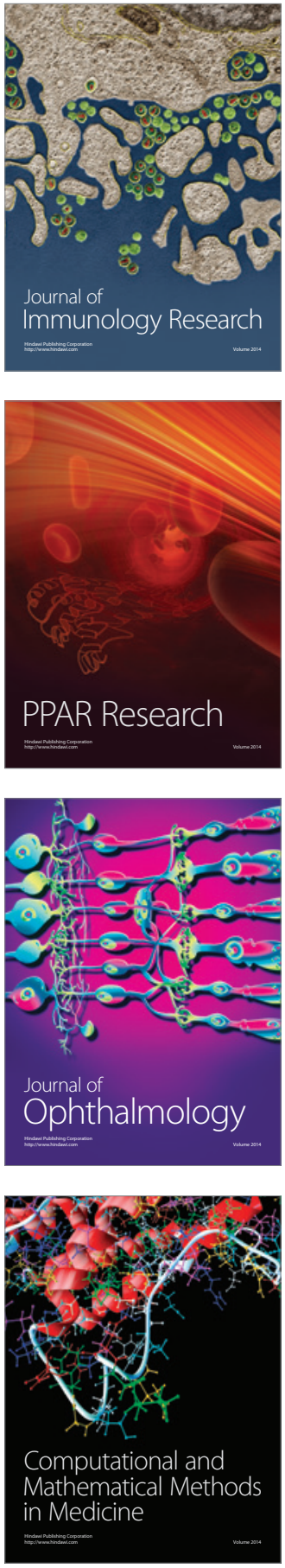

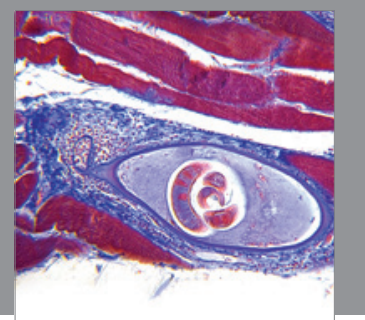

Gastroenterology

Research and Practice
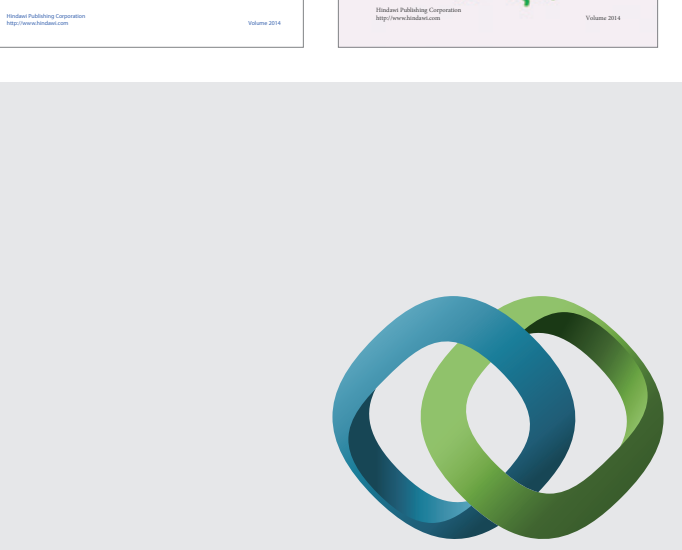

\section{Hindawi}

Submit your manuscripts at

http://www.hindawi.com
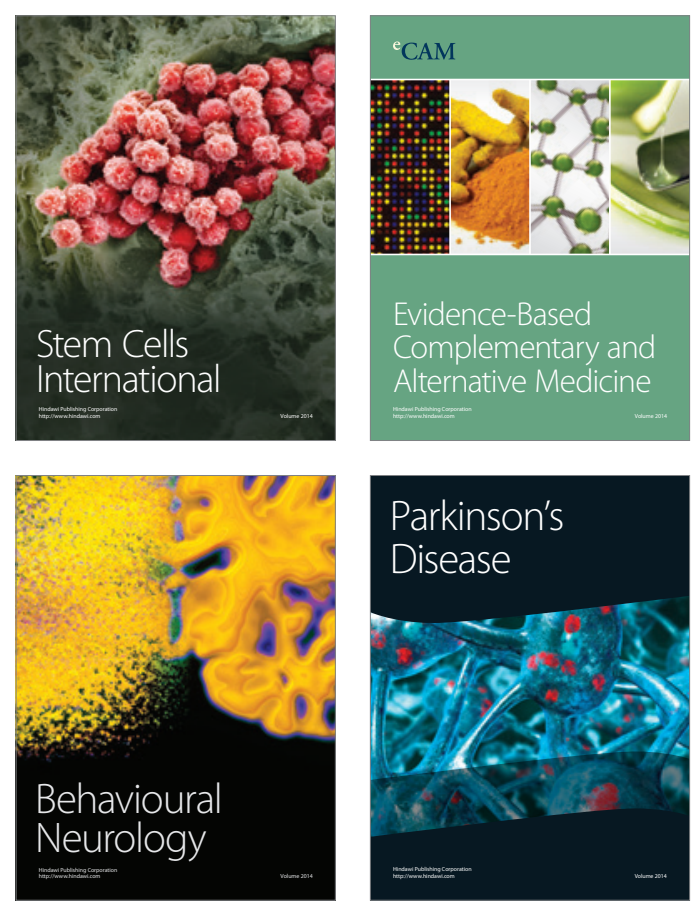

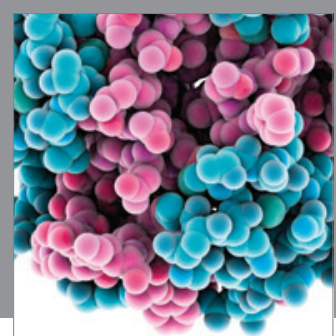

Journal of
Diabetes Research

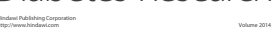

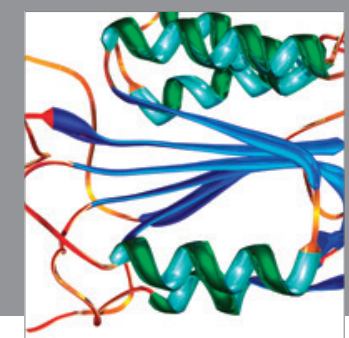

Disease Markers
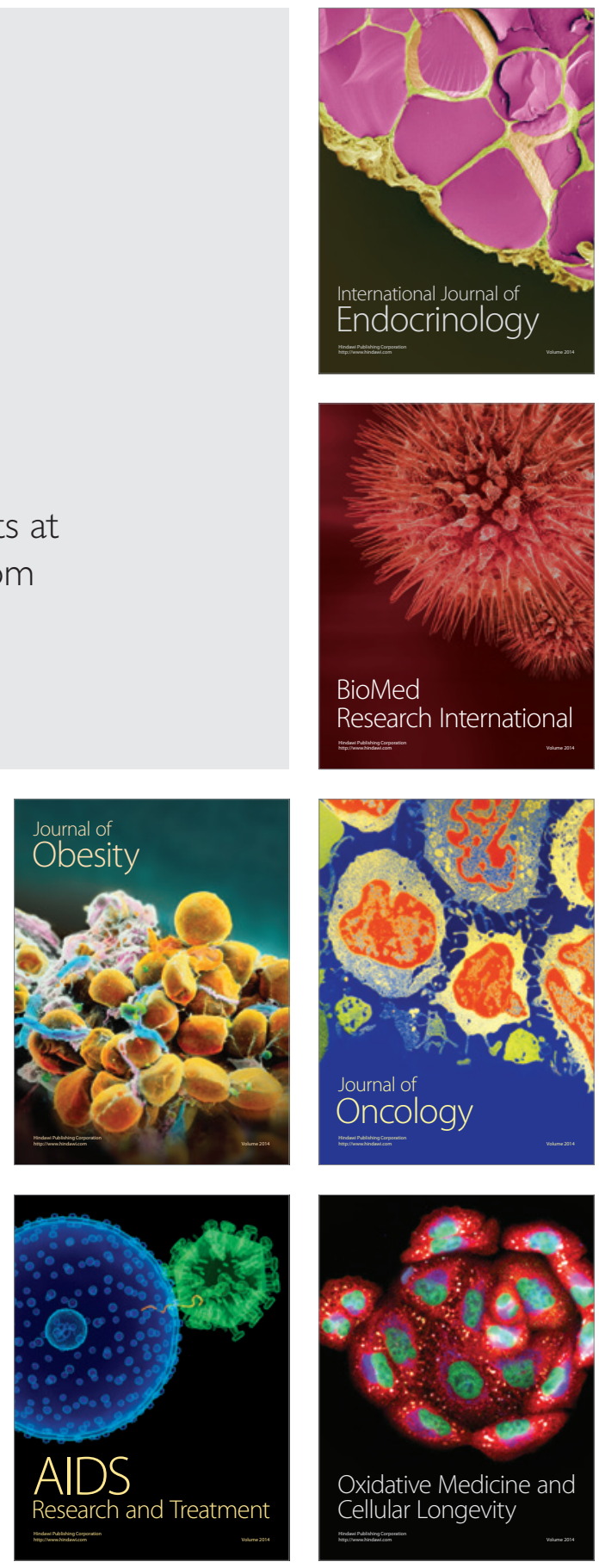